\title{
Clustered Multihop Transmission in Underwater Acoustic Ad-Hoc Networks
}

\author{
Andrej Stefanov and Milica Stojanovic \\ Electrical and Computer Engineering Department \\ Northeastern University \\ E-mail: a.stefanov@neu.edu, millitsa@mit.edu
}

\begin{abstract}
We consider the performance of clustered underwater acoustic ad-hoc networks. We assume a uniform distribution of nodes over a finite area. The nodes in the network form clusters. The information is transmitted across the network from cluster to cluster with nodes in each cluster organized as virtual transmit/receive arrays. The cluster-to-cluster channel is modeled as a Ricean fading channel. We adopt a communication theoretic approach and study the interdependence of the sustainable number of cluster-to-cluster hops through the network, end-toend frame error probability, power and bandwidth allocation. We assume an idealized scenario when there is no interference in the network. We present numerical examples that illustrate the results of the analysis. The results indicate that the network exhibits a bimodal behavior. For a given transmit power, bandwidth and cluster size, a certain minimum network density is required in order to guarantee full network connectivity.
\end{abstract}

\section{INTRODUCTION}

Recently, there has been an increased interest in underwater wireless communications systems [1]. Point-to-point underwater communication systems have been studied extensively [2], [3] resulting in the development of acoustic modem technology [4]. Many applications, however, require an understanding of underwater networking principles. The study of underwater ad-hoc networks is therefore of paramount importance for the design of underwater monitoring sytems. Moreover, driven by the maturation of underwater acoustic modem technology [4], the development of underwater acoustic ad-hoc networks that deliver information where needed is coming close to realization.

In this paper, we consider a network of bottom mounted nodes, hence we focus on a two dimensional network model. We consider a scenario where neighboring nodes in the network are organized into clusters and form virtual transmit/receive arrays [5]. The information is transmitted across the network via multihop cluster-to-cluster routes. We adopt a communication theoretic approach [6] and study the performance of clustered underwater acoustic ad-hoc networks. We investigate the interdependence between the sustainable number of cluster-to-cluster hops in the network as an indicator of network connectivity, as well as power and bandwidth requirements for various network densities. In this study, we consider an idealized scenario in which there is no interference in the network. We find that the network exibits a bimodal behavior; that is, given the available power, bandwidth and cluster size, there is a certain minimum network density that guarantees full network connectivity. Hence, depending on the actual node density, the network will be in one of two regimes (fully connected or not).

The paper is organized as follows. The next section introduces the basic properties of acoustic propagation. The communication theoretic analysis of clustered underwater adhoc networks is presented in Section III. Numerical results illustrating the network behavior are given in Section IV. Section V concludes the paper.

\section{Underwater Acoustic Propagation}

Underwater acoustic communication channels are characterized by a path loss that depends not only on the distance between the transmitter and the receiver, as is the case in many other wireless channels, but also on the signal frequency. The absorption loss increases with frequency, as well as with distance. It imposes a limit on the available bandwidth, due to the practical constraint of finite transmission power.

Attenuation, or path loss, that occurs in an underwater acoustic channel over a distance $d$ for a signal of frequency $f$, is given by

$$
A(d, f)=A_{0} d^{\kappa} a(f)^{d}
$$

where $A_{0}$ is a unit-normalizing constant that also includes fixed losses, $a(f)$ is the absorption coefficient and $\kappa$ is the spreading factor. The spreading factor describes the geometry of propagation and is typically $1 \leq \kappa \leq 2$. In the case of practical spreading, $\kappa=1.5$. The absorption coefficient can be expressed empirically, using Thorp's formula which gives $a(f)$ in $\mathrm{dB} / \mathrm{km}$ for $f$ in $\mathrm{kHz}$ as [2]

$$
10 \log a(f)=\frac{0.11 f^{2}}{1+f^{2}}+\frac{44 f^{2}}{4100+f^{2}}+\frac{2.75 f^{2}}{10^{4}}+0.003
$$

This formula is generally valid for frequencies above a few hundred $\mathrm{Hz}$, which is the usual operating range for the majority of practical systems.

The ambient noise in the ocean can be modeled using four sources: turbulence, shipping, waves and thermal noise. Most of the ambient noise sources can be described by Gaussian statistics and a continuous power spectral density. The following empirical formulae give the power spectral densities of the four noise components in $\mathrm{dB}$ re $\mu \mathrm{Pa}$ per $\mathrm{Hz}$ as a function of frequency in $\mathrm{kHz}$ [2]: 


$$
\begin{aligned}
10 \log N_{\mathrm{t}}(f)= & 17-30 \log f \\
10 \log N_{\mathrm{s}}(f)= & 40+20(s-0.5)+26 \log f \\
& -60 \log (f+0.03) \\
10 \log N_{\mathrm{w}}(f)= & 50+7.5 \sqrt{w}+20 \log f \\
& -40 \log (f+0.4) \\
10 \log N_{\mathrm{th}}(f)= & -15+20 \log f
\end{aligned}
$$

where $s$ is the shipping activity factor and $w$ is the wind speed in $\mathrm{m} / \mathrm{s}$. The overall power spectral density of the ambient noise is

$$
N(f)=N_{\mathrm{t}}(f)+N_{\mathrm{s}}(f)+N_{\mathrm{w}}(f)+N_{\mathrm{th}}(f) .
$$

Using the attenuation $A(d, f)$ and the noise power spectral density $N(f)$ we can evaluate the narrow-band signal-to-noise ratio observed over a distance $d$. The narrow-band signal-tonoise ratio is given by

$$
\operatorname{snr}(d, f)=\frac{S(f)}{A(d, f) N(f)}
$$

where $S(f)$ is the power spectral density of the transmitted signal. The AN product, $A(d, f) N(f)$, determines the frequency dependent part of the signal-to-noise ratio (SNR). The factor $\frac{1}{A(d, f) N(f)}$ is illustrated in Figure 1. For each transmission distance $d$, there clearly exists a frequency $f_{o}(d)$ for which the narrow-band signal-to noise ratio is maximized. In practice, one may choose $f_{o}(d)$ as the operating frequency and allocate a certain transmission bandwidth around it.

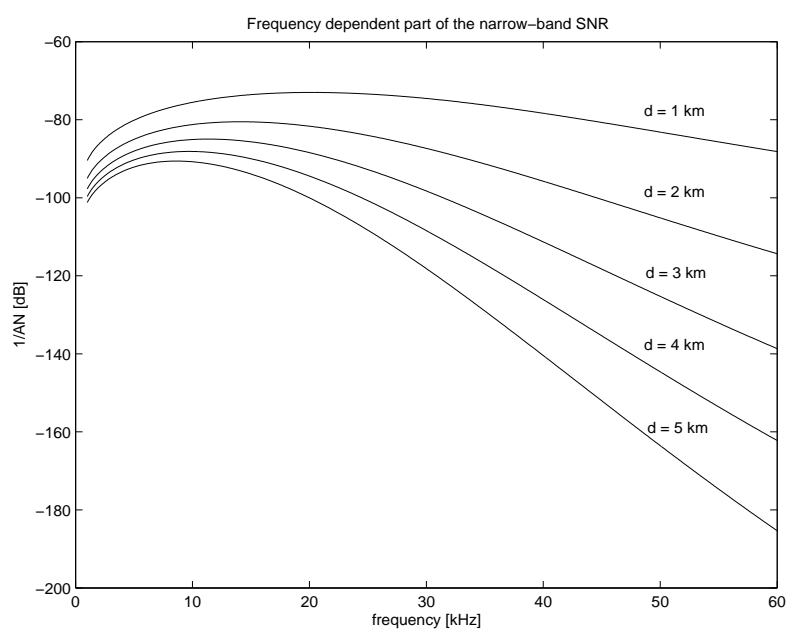

Fig. 1. The AN product for various distances. Spreading factor $\kappa=1.5$.

We define the $3 \mathrm{~dB}$ bandwidth, $B_{3}(d)$, as the range of frequencies around $f_{o}(d)$ for which $A(d, f) N(f)<2 A\left(d, f_{o}(d)\right) N\left(f_{o}(d)\right)$. The operating frequency $f_{o}(d)$ and the $3 \mathrm{~dB}$ bandwidth, $B_{3}(d)$, are shown in Figure 2.

\section{Ad-HOC NeTwORK SETUP}

We consider a network of bottom mounted nodes, i.e., we focus on a two dimensional network that provides coverage

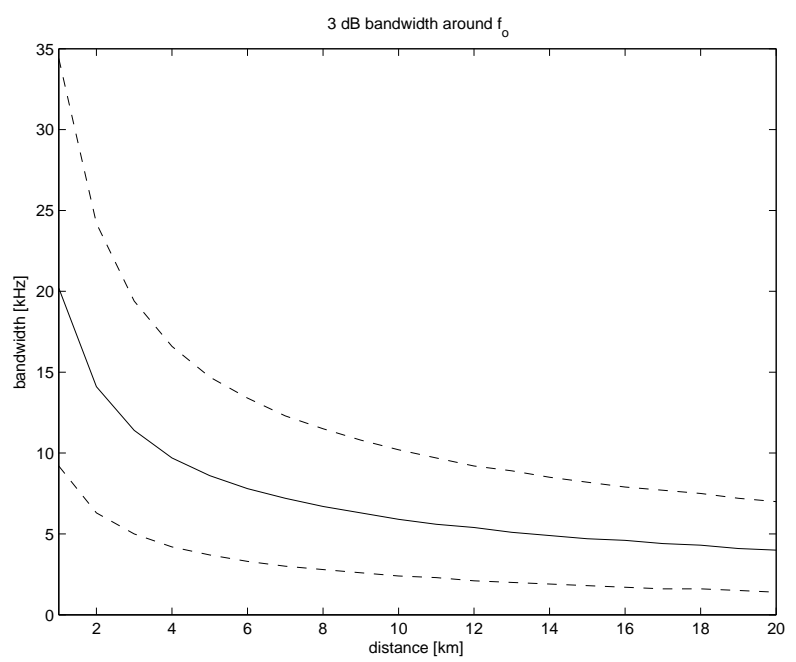

Fig. 2. Frequency $f_{o}(d)$ (solid line), $f_{\max }(d)$ and $f_{\min }(d)$ (dashed lines) which define the $3 \mathrm{~dB}$ bandwidth. Spreading factor $\kappa=1.5$.

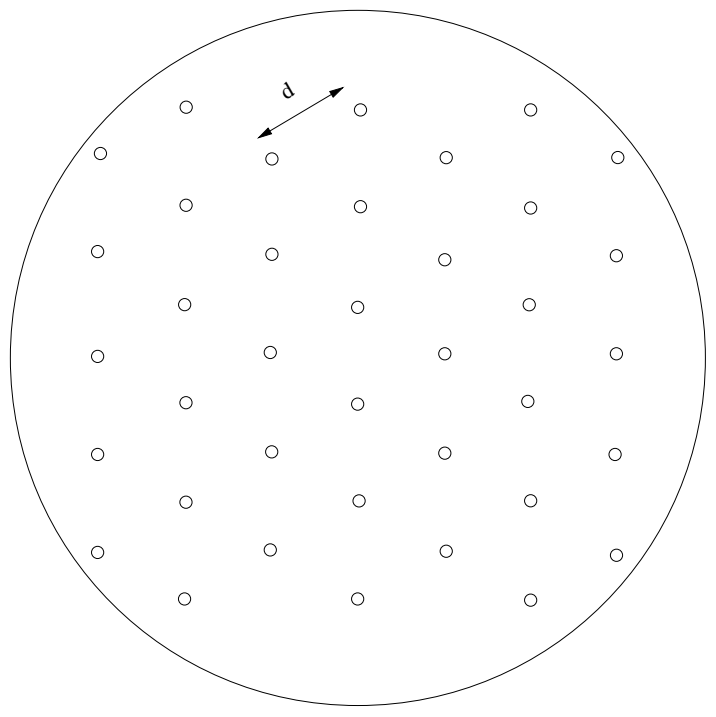

Fig. 3. Uniform network coverage over a circle.

over a certain area. We assume that the area of the network is a circle and consider a uniform distribution of nodes in the network as depicted in Figure 3. Given the total number of nodes in the network, $N$, and the area of the network, $\mathcal{A}$, the density of the network is

$$
\rho_{s}=\frac{N}{\mathcal{A}}
$$

Given the uniform node distribution and circular area of the network, the distance between nodes, $d$, is

$$
d=\frac{c}{\sqrt{\rho_{s}}}
$$

where $c$ is a constant that depends on the node placement (grid pattern). Without loss of generality we assume that $c=1$.

We assume that groups of $N_{c}$ nodes are organized into clusters. We define the distance between clusters as the distance 


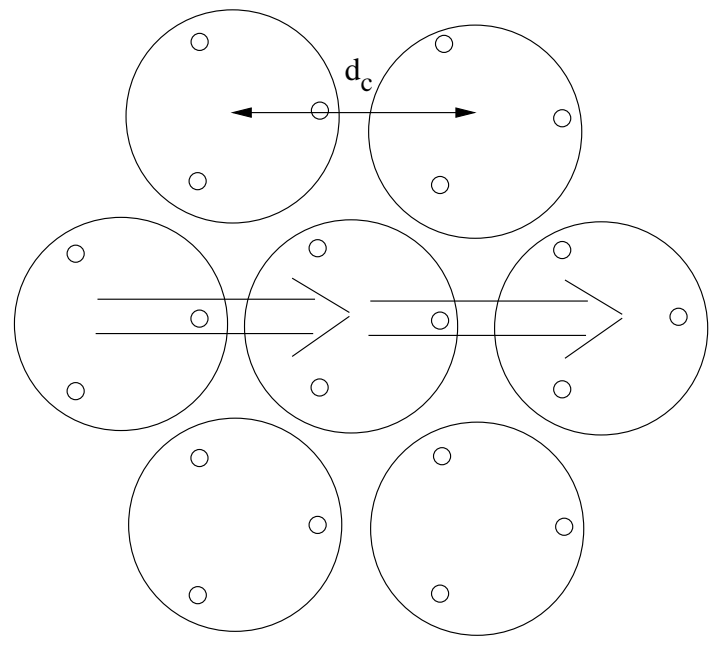

Fig. 4. Cluster-to-cluster transmission with $N_{c}=3$ nodes per cluster

between the centers of the clusters, $d_{c}$. Hence,

$$
d_{c} \approx d \sqrt{N_{c}} \approx \sqrt{\frac{N_{c}}{\rho_{s}}} .
$$

We assume cluster-to-cluster multihop routes along nearest neighbor clusters as depicted in Figure 4. Clustering is an energy saving strategy and as such it may be attractive for networks with limited energy, battery powered nodes. As the longest multihop route in the network is along the diameter of the network, $D$, the maximum number of cluster-to-cluster hops, $n_{h}^{\max }$ is

$$
n_{h}^{\max }=\frac{D}{d_{c}}=\frac{2 \sqrt{\mathcal{A} / \pi}}{\sqrt{N_{c} / \rho_{s}}}=\frac{2}{\sqrt{\pi}} \sqrt{\frac{N}{N_{c}}} .
$$

Let the average number of cluster-to-cluster hops for a multihop route be denoted by $\bar{n}_{h}$. Then, as long as the probability distribution for the number of hops is symmetric we have that [6]:

$$
\bar{n}_{h}=\frac{n_{h}^{\max }}{2}=\frac{1}{\sqrt{\pi}} \sqrt{\frac{N}{N_{c}}} .
$$

We assume a simple distributed space-time block code with a decode-and-forward relaying strategy. The end-to-end frame error probability for a multihop route with $n_{h}$ cluster-to-cluster hops, $p_{\text {route, }}$, is given by

$$
p_{\text {route }}=1-\left(1-p_{b}\right)^{L n_{h}}
$$

where $p_{b}$ denotes the bit error probability of a single clusterto-cluster link and $L$ denotes the frame size in bits.

We consider the quality-of-service for the network in terms of the maximum allowed end-to-end route frame error probability, i.e., we require, $p_{\text {route }} \leq p_{\text {route }}^{\max }$. Let the number of cluster-to-cluster hops that can be sustained by the network, i.e., the number of cluster-to-cluster hops that can satisfy the maximum end-to-end route frame error probability, be denoted by $n_{\mathrm{sh}}$. From Eq. (11), it follows that $n_{\mathrm{sh}}$ can be calculated as

$$
n_{\mathrm{sh}}=\frac{1}{L} \frac{\log \left(1-p_{\text {route }}^{\max }\right)}{\log \left(1-p_{b}\right)} \approx \frac{1}{L} \frac{p_{\text {route }}^{\max }}{p_{b}} .
$$

While the analysis does not consider it explicitly, note that $n_{\text {sh }}$ and $n_{h}^{\max }$ will in practice be chosen as nearest integers. Assuming a Ricean fading model for the cluster-to-cluster channel [7], the bit error probability, $p_{b}$, can be approximated as [8]

$$
p_{b} \lesssim\left(\frac{1+\mathcal{K}}{1+\mathcal{K}+\operatorname{snr}\left(d_{c}, f\right)}\right)^{t r} \exp \left(-\frac{\operatorname{tr} \mathcal{K} \operatorname{snr}\left(d_{c}, f\right)}{1+\mathcal{K}+\operatorname{snr}\left(d_{c}, f\right)}\right)
$$

where $\mathcal{K}$ denotes the Ricean fading factor which we assume to be the same for all node-to-node sub-channels, $t$ denotes the transmit diversity gain and $r$ denotes the receive diversity gain. As an approximation, we assume that the attenuation and the noise are constant over the operational bandwidth, so that the signal-to-noise ratio can be calculated at the operating frequency $f_{o}\left(d_{c}\right)$ as

$$
\operatorname{snr}\left(d_{c}, f_{o}\right)=\frac{P}{A\left(\sqrt{\frac{\mathcal{A} N_{c}}{N}}, f_{o}\right) N\left(f_{o}\right) B}
$$

where $B$ denotes the bandwidth and $P$ denotes the transmit power. We note that this is a suitable approximation for narrow bandwiths. When the bandwidth is wider, the approximation is only valid for a narrow sub-band of the signal. In a multicarrier system, such as OFDM, this can be thought of as the sub-band of one carrier. In that case, the operating frequency $f_{o}\left(d_{c}\right)$ would indicate the performance on one of the carriers. The performance on the other carriers would depend on their respective operating frequency.

In case the $3 \mathrm{~dB}$ definition is used for the bandwidth, rather than a fixed band of frequencies, we can determine the transmission power required to achieve a desired signal-tonoise ratio, $\mathrm{snr}=\mathrm{snr}^{\star}$, as $P=\mathrm{snr}^{\star} \times B_{3}\left(d_{c}\right) A\left(d_{c}, f_{o}\right) N\left(f_{o}\right)$. When the signal power spectral density is constant, $S(f)=S$, we have that

$$
\operatorname{snr}\left(d_{c}, f_{o}\right)=\frac{S}{A\left(\sqrt{\frac{\mathcal{A} N_{c}}{N}}, f_{o}\right) N\left(f_{o}\right)} .
$$

\section{NumericAl RESUlts}

We present numerical examples that illustrate the performance of underwater acoustic clustered ad-hoc networks. We examine the relationships between the sustainable number of cluster-to-cluster hops, the end-to-end frame error probability, the signal power and bandwidth, and the operating frequency. We assume Ricean fading model for each cluster-to-cluster channel. The Ricean fading factor is taken to be $\mathcal{K}=10$. We consider a target (maximum allowed) end-to-end frame error probability (FEP) of $p_{\text {route }}^{\max }=10^{-3}$. We assume a circular network of area $\mathcal{A}=1000 \mathrm{~km}^{2}$. We note that an acoustic signal propagates as a pressure wave whose level is commonly measured in $\mathrm{dB}$ relative to $1 \mu \mathrm{Pa}$. We adopt that convention, 
hence the power levels are expressed in $\mathrm{dB}$ re $\mu \mathrm{Pa}$. We neglect any fixed losses. ${ }^{1}$ The frame size is $L=100$ bits and the cluster size is $N_{c}=3$. The spreading factor is $\kappa=1.5$, the shipping activity factor is $s=0.5$ and we assume calm conditions, that is, the wind speed is $w=0 \mathrm{~m} / \mathrm{s}$.

Figure 5 presents the sustainable number of clusterto-cluster hops for an end-to-end frame error probability of $10^{-3}$, bandwidth $B=4 \mathrm{kHz}$ and transmit power $P=100 \mathrm{~dB}$ re $\mu \mathrm{Pa}$. The average number of cluster-tocluster hops given by Eq. (10) is also presented. The cluster size $N_{c}=3$. We assume that $t=3$ and $r=1$, that is, the nodes in the transmit cluster collaborate to form a distributed space-time code, but a single node acts as a receiver in the receiving cluster. We observe that the sustainable number of hops in the network exhibits a bimodal behavior. Below about $N=300$ nodes, the network cannot sustain routes with an average number of hops. This is due to the fact that with so few nodes in the network, the nodes are too far apart to guarantee the required end-to-end frame error probability for the available transmit power. When the number of nodes in the network is above about 300 nodes, we see that the sustainable number of cluster-to-cluster hops rapidly exceeds the average number of cluster-to-cluster hops. When the number of nodes is $N \gtrsim 300$, we have $n_{\text {sh }} \geq n_{h}^{\max }$. This ensures full connectivity in the network, where all routes satisfy the end-to-end frame error probability requirement. The preferred operating frequency, $f_{o}\left(d_{c}\right)$, is also presented. As the distance between the nodes in the network decreases, the operating frequency, $f_{o}\left(d_{c}\right)$, increases. The signal-to-noise ratio per cluster-to-cluster hop is also presented. The changes in the signal-to-noise ratio are due to the changes in the AN product, which depends on distance and frequency.

Figure 6 depicts the sustainable number of cluster-to-cluster hops for different values of the bandwidth. The end-toend frame error probability is $10^{-3}$ and the transmit power $P=100 \mathrm{~dB}$ re $\mu \mathrm{Pa}$. The cluster size is again $N_{c}=3$. We assume $t=3$ and $r=1$. We observe that the sustainable number of cluster-to-cluster hops decreases as we increase the signal bandwidth. For example, when the bandwidth is $B=1 \mathrm{kHz}$, we have full connectivity for $N \gtrsim 100$ nodes. When the bandwidth is $B=2 \mathrm{kHz}$, we have full connectivity for $N \gtrsim 175$ nodes. If the bandwidth is increased to $B=4 \mathrm{kHz}$, we obtain full connectivity when the number of nodes in the network is $N \gtrsim 300$. Note that this behavior is not inherent to the channel, but is rather a consequence of the fact that we have used the same signal power in all three cases. In other words, while the signal power remains the same, the noise power increases with the increased bandwidth causing an overall degradation in the system performance.

The sustainable number of hops in the case of $3 \mathrm{~dB}$ bandwidth allocation around $f_{o}$ is presented in Figure 7 . The endto-end frame error probability is $10^{-3}$ and we assume constant

\footnotetext{
${ }^{1}$ Inclusion of additional frequency independent losses, and an adjustment of the background noise level to suit a particular environement and provide the necessary SNR margins, will scale the results in absolute value, but will not alter the general behavior.
}
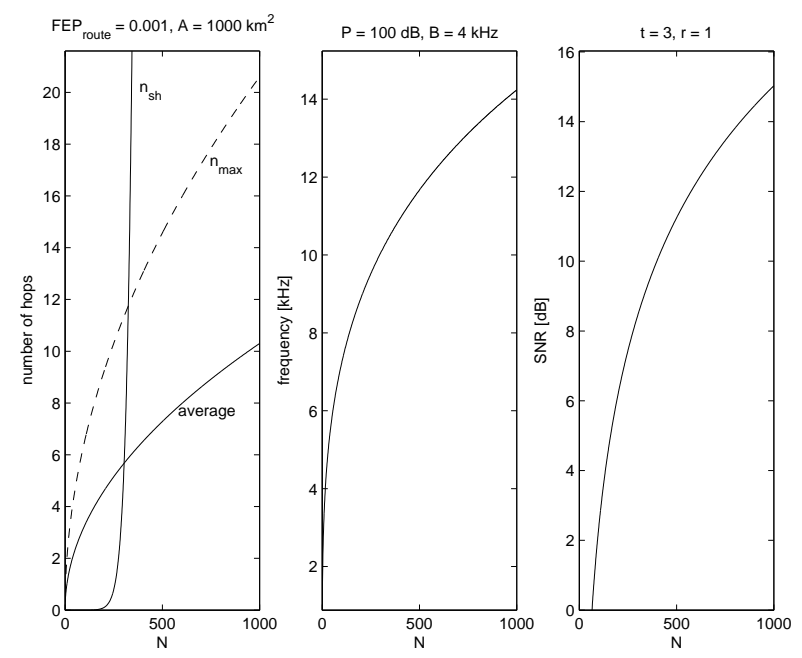

Fig. 5. Sustainable number of cluster-to-cluster hops vs. the number of nodes $N$ for a uniform network with Ricean fading. The network area is $\mathcal{A}=1000 \mathrm{~km}^{2}$, bandwidth $B=4 \mathrm{kHz}$, transmit power $P=100 \mathrm{~dB}$ re $\mu \mathrm{Pa}$, the cluster size $N_{c}=3, t=3, r=1$. The operating frequency $f_{o}\left(d_{c}\right)$ and SNR are also presented.

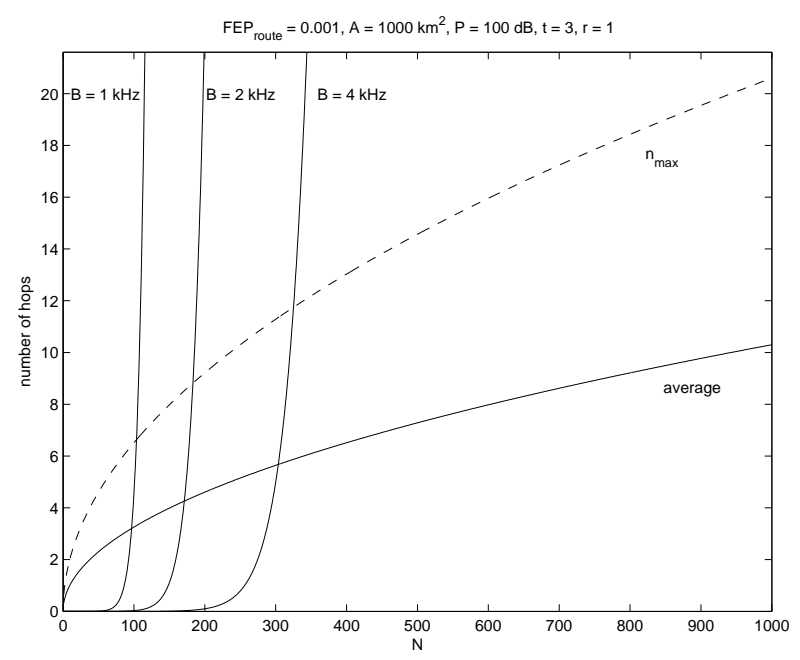

Fig. 6. Sustainable number of cluster-to-cluster hops vs. the number of nodes $N$ for a uniform network with Ricean fading. The network area is $\mathcal{A}=1000 \mathrm{~km}^{2}$, transmit power $P=100 \mathrm{~dB}$ re $\mu \mathrm{Pa}$, the cluster size $N_{c}=3, t=3, r=1$.

transmit power spectral density $S=95 \mathrm{~dB}$ re $\mu \mathrm{Pa}$ per $\mathrm{Hz}$ for $f$ in $\mathrm{kHz}$. The cluster size $N_{c}=3, t=3$ and $r=1$. We also present $f_{o}$ and the $3 \mathrm{~dB}$ bandwidth. We observe that the $3 \mathrm{~dB}$ bandwidth increases as the number of nodes in the network increases. This is due to the fact that the distance between nodes in the network decreases as $N$ increases, and as indicated in Figure 1, as the distance decreases, $\frac{1}{A(d, f) N(f)}$, increases and becomes smoother.

So far, we have assumed mulihop transmissions in which neighboring clusters are always connected. It is also possible to consider a situation in which direct links are formed between non-neighboring clusters. The motivation for doing so is interference avoidance, as illustrated in the next example. 

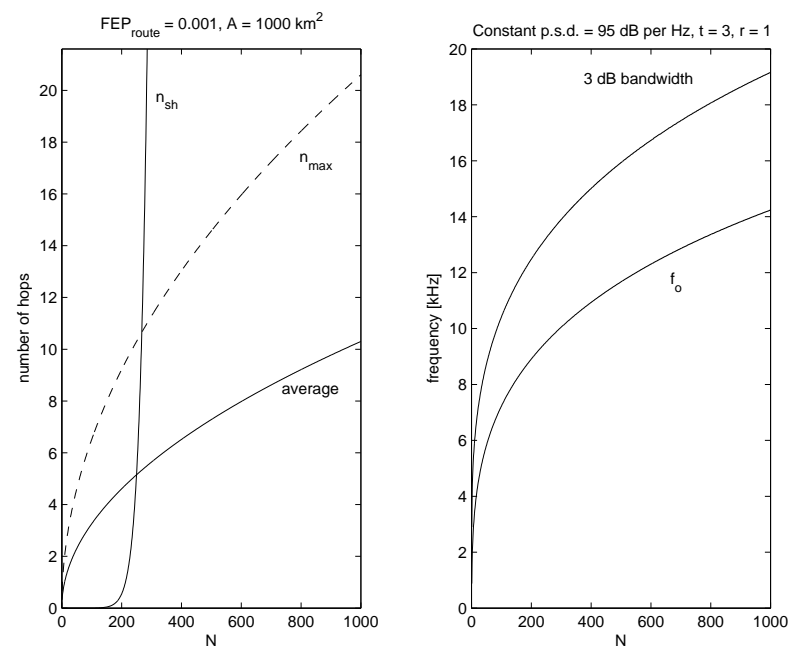

Fig. 7. Sustainable number of cluster-to-cluster hops vs. the number of nodes $N$ for a uniform network with Ricean fading. The network area is $\mathcal{A}=1000 \mathrm{~km}^{2}, 3 \mathrm{~dB}$ bandwidth $B_{3}\left(d_{c}\right), S=95 \mathrm{~dB}$ re $\mu \mathrm{Pa}$ per $\mathrm{Hz}$, the cluster size $N_{c}=3, t=3, r=1$.

In this scenario, the cluster-to-cluster distance generalizes to

$$
d_{c} \approx m \sqrt{\frac{N_{c}}{\rho_{s}}}
$$

where $m$ is an integer indicating the number of clusters that are contained in one hop. The average number of cluster-tocluster hops then generalizes to

$$
\bar{n}_{h}=\frac{1}{m \sqrt{\pi}} \sqrt{\frac{N}{N_{c}}} .
$$

Figure 8 presents the sustainable number of cluster-to-cluster hops for an end-to-end frame error probability of $10^{-3}$, bandwidth $B=4 \mathrm{kHz}$, transmit power $P=110 \mathrm{~dB}$ re $\mu \mathrm{Pa}$, when the cluster-to-cluster hops are performed across $m=2$ clusters. The average number of cluster-to-cluster hops corresponding to this scenario is also presented. In comparison to the first example, where $m=1$, we observe that as we hop across an increased distance the required power has increased. Of course, the average number of hops is reduced. The preferred operating frequency is also reduced. For example when there are $N=1000$ nodes in the network, $f_{o}\left(d_{c}\right)=14 \mathrm{kHz}$ when $m=1$, (see Figure 5), but when $m=2$, we have $f_{o}\left(d_{c}\right)=9.5 \mathrm{kHz}$. Hence, in this scenario, as long as the bandwidth centered around the respective operating frequency $f_{o}\left(d_{c}\right)$ is approximately $B \leq 4 \mathrm{kHz}$, the routes through the network with $m=1$ would not create interference for the routes through the network for which $m=2$ and vice versa.

\section{CONCLUSIONS}

We considered a two dimensional network of bottom mounted nodes. The nodes were organized as clusters forming virtual transmit/receive arrays. The cluster-to-cluster channel was modeled as a Ricean fading channel. We studied the sustainable number of cluster-to-cluster hops through the network
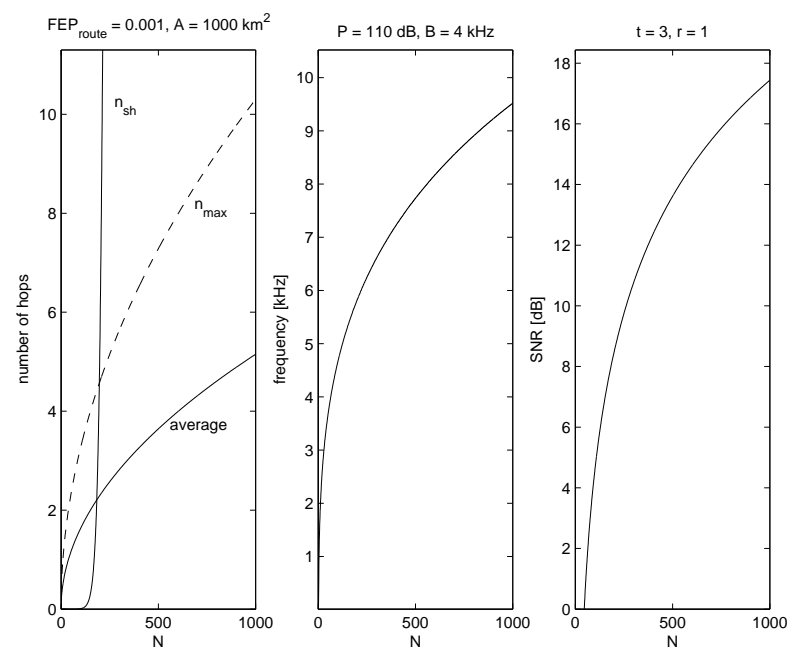

Fig. 8. Sustainable number of cluster-to-cluster hops vs. the number of nodes $N$ for a uniform network with Ricean fading. The network area is $\mathcal{A}=1000 \mathrm{~km}^{2}$, bandwidth $B=4 \mathrm{kHz}$, transmit power $P=110 \mathrm{~dB}$ re $\mu \mathrm{Pa}$, the cluster size $N_{c}=3, m=2, t=3, r=1$. The operating frequency $f_{o}\left(d_{c}\right)$ and SNR are also presented.

as an indicator of network connectivity and its dependence on the power and bandwidth allocation in an idealized scenario when there is no interference in the network. We found that the sustainable number of hops in the network exhibits a bimodal behavior: as long as the number of nodes is greater than some minimum, connectivity is guaranteed; otherwise, packet transmissions meeting a certain quality-of-service requirement, e.g., a target FEP, cannot be guaranteed across the entire network diameter. This is due to the fact that with so few nodes in the network, the nodes are too far apart to guarantee the required end-to-end frame error probability for the available transmit power.

\section{ACKNOWLEDGMENTS}

This work was supported in part by the NSF grant 0831728 and the ONR grant N00014-09-1-0700.

\section{REFERENCES}

[1] IEEE Journal on Selected Areas in Communications, Special Issue on Underwater Wireless Communications and Networks, December 2008.

[2] M. Stojanovic and J. Preisig, "Underwater Acoustic Communication Channels: Propagation Models and Statistical Characterization," IEEE Communications Magazine, pp. 84-89, January 2009.

[3] M. Stojanovic, "On the Relationship Between Capacity and Distance in an Underwater Acoustic Channel," ACM SIGMOBILE Mobile Comp. Commun. Rev., vol. 11, No. 4, pp. 34-43, October 2007.

[4] L. Freitag et al., "The WHOI Micro-Modem: An Acoustic Communications and Navigation System for Multiple Platforms," in Proc. IEEE Oceans Conference, 2005.

[5] M. Dohler, "Virtual Antenna Arrays," Ph.D. Thesis, King's College, London, UK, 2003.

[6] O. Tonguz and G. Ferrari, "Ad Hoc Wireless Networks: A Communication-Theoretic Perspective," Wiley, 2006.

[7] P. Qarabaqi and M. Stojanovic, "Statistical Modeling of a Shallow Water Acoustic Communication Channel," in Proc. Underwater Acoustic Measurements Conference, Nafplion, Greece, June, 2009.

[8] S. Benedetto and E. Biglieri, "Principles of Digital Transmission with Wireless Applications,” Kluwer/Plenum, 1999. 